\title{
Devers-Kanoglu, Ulrike
}

Informelles Lernen in kommunalen Partnerschaften zwischen Nord und Süd

- lokal und global?

ZEP : Zeitschrift für internationale Bildungsforschung und Entwicklungspädagogik 30 (2007) 4, S. $13-16$

Quellenangabe/ Reference:

Devers-Kanoglu, Ulrike: Informelles Lernen in kommunalen Partnerschaften zwischen Nord und Süd lokal und global? - In: ZEP : Zeitschrift für internationale Bildungsforschung und

Entwicklungspädagogik 30 (2007) 4, S. 13-16 - URN: urn:nbn:de:0111-opus-60878 - DOI:

10.25656/01:6087

https://nbn-resolving.org/urn:nbn:de:0111-opus-60878

https://doi.org/10.25656/01:6087

in Kooperation mit / in cooperation with:

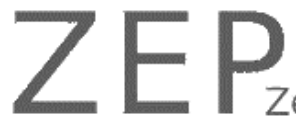

Zeitschrift für internationale Bildungsforschung und Entwicklungspädagogik

"Gesellschaft für interkulturelle Bildungsforschung und Entwicklungspädagogik e.V."

http://www.uni-bamberg.de/allgpaed/zep-zeitschrift-fuer-internationale-bildungsforschung-und-entwicklungspaedagogik/profil

\section{Nutzungsbedingungen}

Gewährt wird ein nicht exklusives, nicht übertragbares, persönliches und beschränktes Recht auf Nutzung dieses Dokuments. Dieses Dokument ist ausschließlich für den persönlichen, nicht-kommerziellen Gebrauch bestimmt. Die Nutzung stellt keine Übertragung des Eigentumsrechts an diesem Dokument dar und gilt vorbehaltlich der folgenden Einschränkungen: Auf sämtlichen Kopien dieses Dokuments müssen alle Urheberrechtshinweise und sonstigen Hinweise auf gesetzlichen Schutz beibehalten werden. Sie dürfen dieses Dokument nicht in irgendeiner Weise abändern, noch dürfen Sie dieses Dokument für öffentliche oder

kommerzielle Zwecke vervielfältigen, öffentlich ausstellen, aufführen, vertreiben oder anderweitig nutzen.

Mit der Verwendung dieses Dokuments erkennen Sie die Nutzungsbedingungen an.

\section{Terms of use}

We grant a non-exclusive, non-transferable, individual and limited right to using this document.

This document is solely intended for your personal, non-commercial use. Use of this document does not include any transfer of property rights and it is conditional to the following limitations: All of the copies of this documents mus retain all copyright information and other information regarding legal protection. You are not allowed to alter this document in any way, to copy it for public or commercial purposes, to exhibit the document in public, to perform, distribute or otherwise use the document in public.

By using this particular document, you accept the above-stated conditions of use.

\section{Kontakt / Contact:}

peDOCS

DIPF | Leibniz-Institut für Bildungsforschung und Bildungsinformation

Informationszentrum (IZ) Bildung

E-Mail:pedocs@dipf.de

Internet: www.pedocs.de

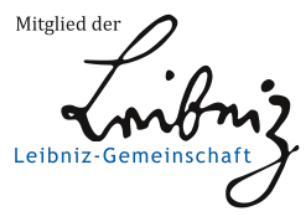



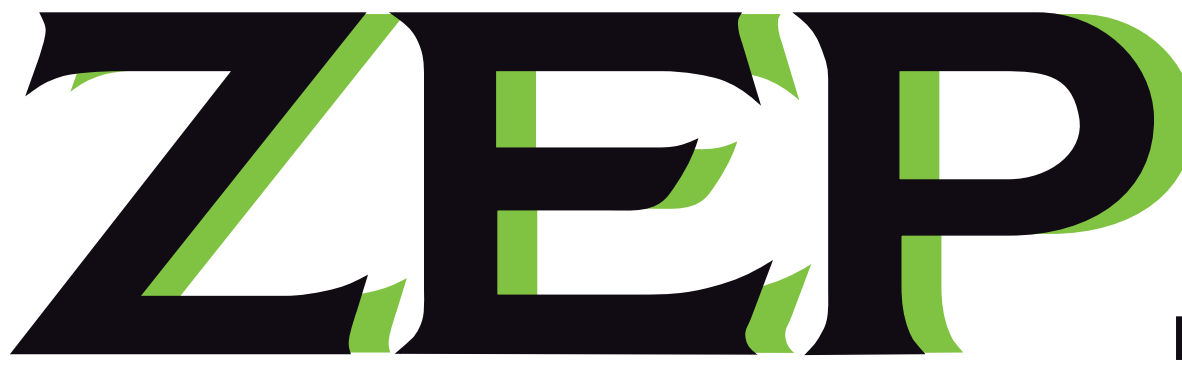

Zeitschrift für internationale Bildungsforschung und Entwicklungspädagogik 30. Jahrgang - Heft 4 - 2007 - ISSN 1434-4688 D - Preis: 6,00€

Mit: Mitteilungen der DGfE-Kommission Vergleichende und Internationale Erziehungswissenschaft

\section{Informelles Lernen und Bildung für nachhaltige Entwicklung}
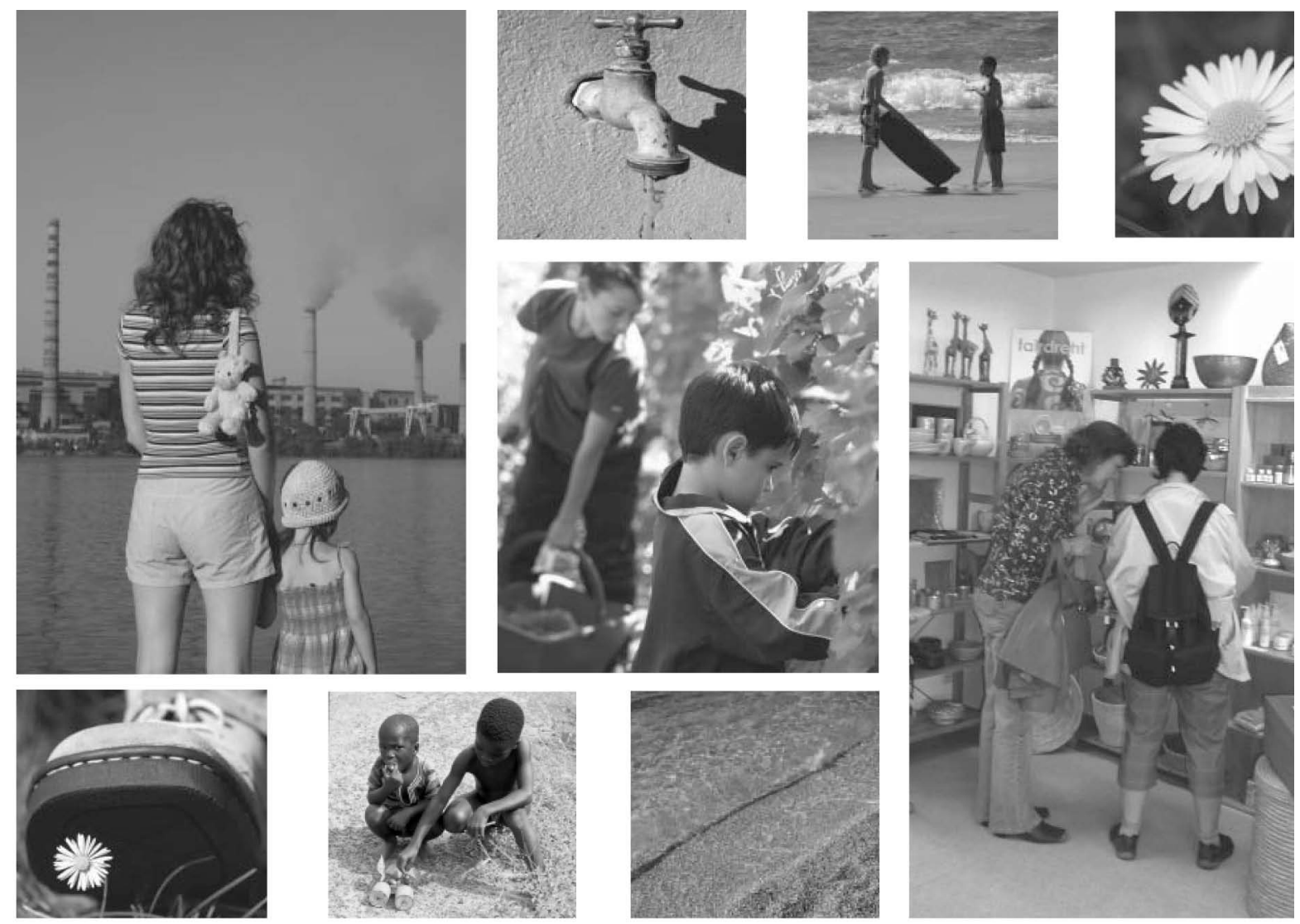

\section{Aus dem Inhalt:}

- Informelles Lernen in der beruflichen Bildung

- Informelles Lernen an der Sustainable University

- Informelles Lernen in kommunalen Nord-Süd-Partnerschaften

- Lernpotenziale im Kontext freiwilligen Engagements von Jugendlichen

- Interventionen in (Bildungs-)Systeme 


\section{Zeitschrift für internationale Bildungsforschung und Entwicklungspädagogik}

30. Jahrgang

I. Frost/W. Helmeth/M. Rohs

M. Adomßent/

G. Michelsen/

M. Rieckmann/

U. Stoltenberg

Ulrike DeversKanoglu

Wiebken Düx/ Erich Sass

Kompetenzerwerb Jugendlicher durch ein freiwilliges Engagement

Manfred Wallenborn

Interventionen in (Bildungs-)Systeme durch die Entwicklungszusammenarbeit. Ein Beitrag zur Wirkungsdebatte

Porträt

Globales Lernen in der Diskussion/Die Erd-Charta in der Schule einsetzen/ 4. BREBIT/Diskussion um Bildungsentwicklung/„Am Ball bleiben für eine zukunftsfähige Welt von morgen" $/$, Respekt! - Youth for peace $\% /$, Eine Welt" für die Klassenstufe 1 bis 10

36 Rezensionen

40 Informationen

\section{Impressum}

ZEP - Zeitschrift für internationale Bildungsforschung und Entwicklungspädagogik 30. Jg. 2007, Heft 4

Herausgeber: Gesellschaft für interkulturelle Bildungsforschung und Entwicklungspädagogik e.V. und KommEnt

Schriftleitung: Annette Scheunpf ug

Redaktionsanschrift: ZEP-Redaktion, Pädagogik I, EWF, Regensburger Str. 160, 90478 Nürnberg

Verlag: Verlag für Interkulturelle Kommunikation (IKO), Postfach 9004 21, 60444 Frankfurt/ Main, Tel.: 069/784808; ISSN 1434-4688 D

Erscheinungsweise und Bezugsbedingungen: erscheint vierteljährlich; Jahresabonnement EUR 20,- Einzelheft EUR 6,-; alle Preise verstehen sic zuzüglich Versandkosten; zu beziehen durch alle Buchhandlungen oder direkt vom Verlag. Abbestellungen spätestens acht Wochen vor Ablauf des Jahres.
Redaktion: Barbara Asbrand, Hans Bühler, Asit Datta, Heidi Grobbauer (Österreich), Helmuth Hartmeyer (Österreich), Richard Helbling (Schweiz), Linda Helfrich, Torsten Jäger, Ulrich Klemm, Gregor Lang-Wojtasik, Volker Lenhart, Claudia Lohrenscheit, Bernd Over wien, Georg-Friedrich Pfäff in, Annette Scheunpf ug, Birgit Schößwender, Klaus Seitz, Barbara Toepfer Technische Redaktion: Claudia Bergmüller (verantwortlich; Rezensionen) 0911/5302-735, Sarah Lange, Christine Schmidt (Infos) Abbildungen: (Falls nicht bezeichnet) Privatfotos oder Illustrationen der Autoren.

Titelbild: (C) vgl. www.fotolia.com

Diese Publikation ist gefördert vom Evangelischen Entwicklungsdienst-Ausschuss für Entwicklungsbezogene Bildung und Publizistik, Bonn. Das Heft ist auf umweltfreundlichem chlorfreien Papier gedruckt. 


\section{Ulrike Devers-Kanoglu}

\section{Informelles Lernen in}

\section{kommunalen Partnerschaften} zwischen Nord und Süd - lokal und global?

Zusammenfassung: Die Autorin beschreibt kommunale Partnerschaften zwischen Nord und Süd, die sich im Kontext der (Lokalen) Agenda 21 verorten und dabei verschiedene Akteure auf kommunaler Ebene integrier en, als mögliches Feld informellen Lernens im Sinne einer nachhaltigen Entwicklung. Dabei werden sowohl die darin liegenden Chancen als auch die damit verbundenen Herausfor derungen in den Blick genommen.

Abstract: The author describes partnerships between Northern and Southern municipalities as environments that provide options for informal learning in the feld of sustainable development, especially when they ar e rooted in the context of (Local) Agenda 21 and based on partnerships at a local level. Thereby, the inherent chances are considered as well as the challenges.

\section{Kommunale Partnerschaften zwischen Nord und Süd im Kontext der (Lokalen) Agenda 21}

Kommunale Kooperationsbeziehungen zwischen Nord und Süd sind so vielgestaltig wie dieAkteure, die derartige Kooperationen mit Leben füllen. ${ }^{1}$ Um dieses weite Feld gemäß der Intention der vorliegenden Veröffentlichung einzugrenzen, möchte ich mich im Folgenden auf Kooperationen und Partnerschaften zwischen Kommunen in Nord und Süd beschränken, die sich thematisch im Bereich der (Lokalen) Agenda 21 verorten und verschiedeneAkteure auf kommunaler Ebene integrieren. ${ }^{2}$ Diese fühlen sich vielfach nicht allein einer wie auch immer gearteten Kooperation, sondern explizit auch dem lernenden Austausch zwischen den Kooperationspartnern unter der gemeinsamen Perspektive einer nachhaltigen Entwicklung verpf ichtet. Dies wird unter anderem in Zielformulierungen deutlich, welche z.B. das , Voneinander Lernen' in den Vordergrund stellen. Erwartungen an derartige Kooperationen implizieren demgemäß häuf g ein informelles, erfahrungsbasiertes, kooperatives
Lernen im Zuge partnerschaftlicher Kooperation: „Durch Erfahrungsaustausch und Zusammenarbeit zwischen Süd und Nord“ - so eine der Vermutungen - „können Modelle eines ökologisch nachhaltigen und sozial verträglichen Stadtlebens gemeinsam entwickelt werden." ${ }^{3}$ Weitere Erwartungen gehen dahin, dass kommunale Kooperationen

- ,globale Zusammenhänge aufzeigen, Bewusstsein vor Ort für eine zukunftsfähige Entwicklung schaffen und gemeinsame Lernprozesse fördern;

- persönliche Kontakte und konkrete Begegnungen ermöglichen, die zum Abbau von Vorurteilen und zur Motivation der Einzelnen und ihrer Handlungsbereitschaft beitragen;

- (...)

- gemeinsam mit ihren Akteursgruppen, Bürgerinitiativen und BürgerInnen Lösungskompetenz für globalisierte Prozesse in wichtigen gesellschaftlichen Bereichen entwickeln." ${ }^{\text {"4 }}$

Die Besonderheit des hier umrissenen Handlungsfeldes liegt auf der Hand: das - noch näher zu beschreibende - Lernen der Akteure ist zunächst primär in den jeweiligen kommunalen Kontexten verankert; in diesen interagieren unterschiedliche Akteure unter Berücksichtigung lokal relevanterThematiken. Durch die Kooperationsbeziehung mit einer Partnerkommune im Ausland kommt jedoch noch ein weiterer zentralerAspekt hinzu: der themenbezogene Austausch der Akteure mit den jeweiligen Partnern. ${ }^{5}$

\section{Informelles Lernen in kommunalen} Partnerschaften zwischen Nord und Süd

Partnerschaften dieser Art werden gemäß der vorangehenden Ausführungen als Feld kooperativen Handelns begriffen, in welchem bei den beteiligtenAkteuren im Zuge des Engagements Lern- und Bildungsprozesse im Sinne einer nachhaltigen Entwicklung angeregt werden können. 


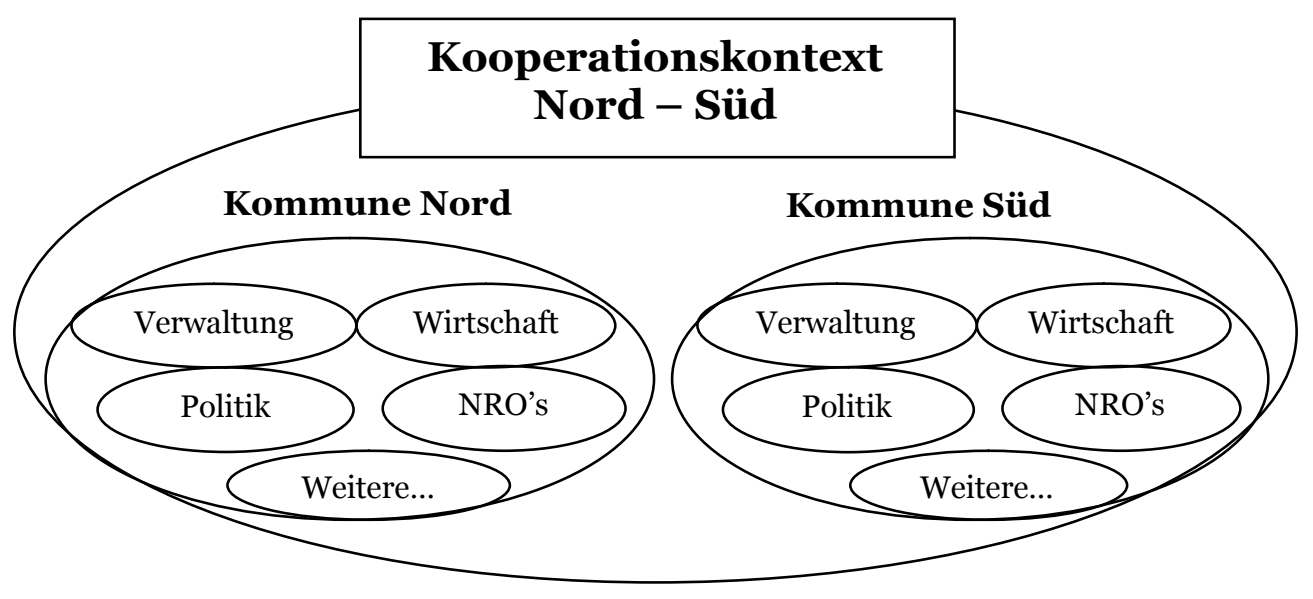

Abb. 1: Kontexte informellen Lernens in kommunalen Partnerschaften

Im Folgenden soll entsprechend der Struktur dieses Feldes ein besonderes Augenmerk auf das informelle Lernen der Akteure gelegt werden. Dieses wird hier gemäß D£ nition der Europäischen Kommission verstanden als ein „Lernen, das im Alltag, am Arbeitsplatz, im Familienkreis oder in der Freizeit statt $f$ ndet. Es ist (in Bezug auf Lernziele, Lernzeit oder Lernförderung) nicht strukturiert und führt üblicherweise nicht zur Zertif zierung. Informelles Lernen kann zielgerichtet sein, ist jedoch in den meisten Fällen nichtintentional (oder inzidentell/beiläuf g). ${ }^{7}$

Der Kontext des informellen Lernens in derartigen Partnerschaften erweist sich bei näherer Betrachtung als äußerst komplex. Bereits die intrakommunale Kooperation zwischen den beteiligten Akteuren vor Ort kann vielfältige Möglichkeiten im Hinblick auf informelles Lernens im Sinne einer nachhaltigen Entwicklung bieten. ${ }^{8}$ Dieses ist - so steht zu vermuten - mit den jeweiligen lokalen Bedingungen verwoben, basiert auf lokal relevantemWissen bzw. lokalen Relevanzen und besitzt zudem vor Ort einen unmittelbaren Handlungs- und Anwendungsbezug. Dergleichen vollzieht sich analog in der Partnerkommune. Zwischen beiden vollzieht sich dann die ,eigentliche' interkommunale Kooperation, wie im Folgenden grob schematisch dargestellt:

Dabei stellen grundlegende lebensweltliche und kulturelle Gemeinsamkeiten innerhalb der jeweiligen kommunalen Kontexte - davon ist trotz aller Unterschiedlichkeiten der Gruppierungen und Akteure vor Ort auszugehen - einen Rahmen für das informelle Lernen derAkteure dar, welcher diesen fraglos gegeben ist, sprich: kaum bewusst und damit auch kaum ref ektiert wird. ${ }^{9}$ Durch den Kooperationskontext Nord - Süd kommt eine zusätzliche Qualität hinein: die Option nämlich, in derinterkommunalen Kooperation eben jene Dimension des ,Nicht-mehrHinterfragten' zum Gegenstand der Auseinandersetzung zu machen und so weiterführende Lern- und Bildungsprozesse anzustoßen.

So können z.B. politische Teilhabekompetenzen durch lokales Engagement und Partizipation vor Ort entwickelt werden ${ }^{10}$,wenn beispielsweise durch die Kooperation mit den Partnern der jeweils anderen Kommune auch die Kooperation innerhalb des eigenen Gemeinwesens an handlungspraktischer Relevanz gewinnt. Gleichzeitig kann die Begegnung und Kooperation mit den jeweiligen Partnern aber auch jene anregende oder widerständige Funktion einnehmen, durch welche den beteiligtenAkteuren bislang Implizites und nicht Hinterfragtes aus den jeweiligen alltagsweltlichen Bezügen zu Bewusstsein kommt und ggfs. neu mit den Partnern verhandelt werden muss. ${ }^{11}$

Dabei können die jeweiligen Kooperationspartner - in je unterschiedlichem Ausmaß und abhängig von den intendierten oder inzidentell auftretenden Gegenständen - implizite oder explizite Lehr- und Lernfunktionen einnehmen, was dazu führt, dass die Lehr-Lern-Relationen im Sinne eines , Von- und Miteinander Lernens' hochgradig komplex sind. So werden z.B. in Kooperationsprojekten, Austauschprogrammen u.ä. immer auch gezielt Informationen weiter gegeben, bzw. gezielt Informationen gesucht oder sogar Trainingsprogramme angeboten ${ }^{12}$. Andererseits vollziehen sich gerade neben diesen intentionalen Lehr - und Lernbestrebungen auch stark inzidentelle Formen des Lernens im Zuge von Begegnungen und konkreten Erfahrungen. ${ }^{13}$

\section{Kommunale Partnerschaften - Optionen für informelles Lernen im Sinne einer nachhaltigen Entwicklung}

Die thematische Anbindung kommunaler Partnerschaften an die (Lokale) Agenda 21 alleine garantiert aber noch nicht, dass das informelle Lernen der beteiligten Akteure Relevanz für Lern- bzw. Bildungsprozesse im Sinne einer nachhaltigen Entwicklung besitzt. Diverse Publikationen über Partnerschaften zwischen Kommunen sowie Nichtregierungsorganisationen in Nord und Süd betonen neben den Chancen derartiger Kooperationsbeziehungen auch Hindernisse, die es zu überwinden gilt, so beispielsweise Machtasymmetrien zwischen den Partnern. ${ }^{14}$ Den Inten- 


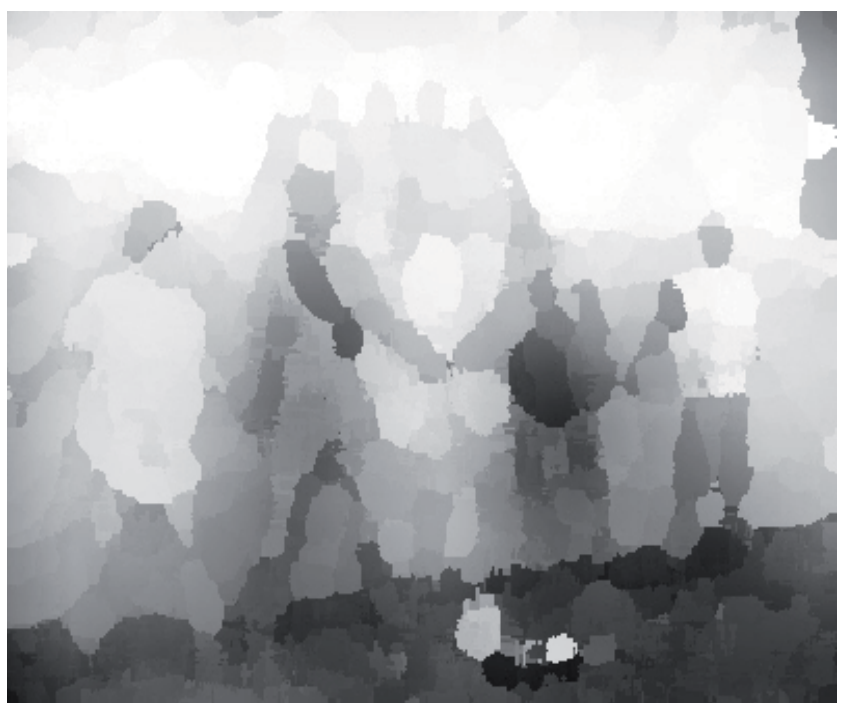

Foto: Uta Göbel-Groß

tionen einer nachhaltigen Entwicklung zuwiderlaufend können diese dazu führen, dass anstelle einer Verständigung über nachhaltige Entwicklung neue Abhängigkeitsund Dominanzverhältnisse sowie Frustrationen bei den Beteiligten entstehen.

Anders gewendet liegen aber gerade in der Dif ferenz besondere Optionen für informelles Lernen im Sinne einer nachhaltigen Entwicklung. Die Unterschiedlichkeit der Kooperationspartner ${ }^{15}$ bietet vielfältige Möglichkeiten - und vielfach auch die Notwendigkeit - das je eigeneVerständnis von nachhaltiger Entwicklung sowie die diesemVerständnis zugrunde liegenden Bedingungen in der Begegnung und Auseinandersetzung mit den Partnern zu re f ektieren. Die komplexe Herausforderung im Sinne einer nachhaltigen Entwicklung liegt somit auch darin begründet, eben diese Bedingungen - lernend - aufzuklären und im Weiteren zu gestalten. ${ }^{16}$

Neben Möglichkeiten des Erwerbs konkreter Fähigkeiten bzw. konkreten Anregungen für die Gestaltung kommunal nachhaltiger Entwicklung im Austausch mit den Partnern besitzt dieses Feld informellen Lernens durch eben jene ref exiven Anteile im Vergleich und in der Aus-



Foto: Uta Göbel-Groß einandersetzung mit den Partnern viel versprechende Optionen für die Entwicklung von Kompetenzen im Sinne einer nachhaltigen Entwicklung ${ }^{17}$ : so beispielsweise im Hinblick auf die ,Kompetenz für transkulturelle Verständigung und Kooperation' sowie die ,Kompetenz zur distanzierten Ref exion über individuelle wie kulturelle Leitbilder'. Aber auch die ,Fähigkeit zu interdisziplinären Herangehensweisen' sowie die ,Fähigkeit zu Engagement und Solidarität' u.a. können in derart umfassenden und über greifenden Kooperationskontexten gefördert werden.

\section{Resümee}

Partnerschaften zwischen Nord und Süd können - so die hier verfolgte Argumentationslinie - einen ausgesprochen fruchtbaren Kontext informellen Lernens im Sinne einer nachhaltigen Entwicklung darstellen. In diesem werden zunächst die in der Regel separat betrachteten ,Bereiche' des informellen Lernens zusammengeführt, wie z.B. infor melles Lernen in beruf ichen Kontexten, im Ehrenamt, in der Freizeit etc., indem sich diese im partnerschaftlichen Austausch vielfach vermischen. Gleichzeitig stellen sie den Versuch dar, verschiedene kulturelle Kontexte in einer übergreifenden Lernkultur zu verbinden, welche lokal ausgerichtete und gleichzeitig weltgesellschaftliche Intentionen hat. Dieser Spagat kann nicht nur dazu beitragen, dass Menschen im Zuge der Kooperation sehr konkrete Dinge lernen, sondern angesichts widerständiger Situationen auch eine (weiterführende) Ref exion des bislang innerhalb der jeweiligen Lernkulturen unhinterfragt Gegebenen und Tradierten anregen.

\section{Anmerkungen}

1 Einen Überblick über Kooperationsbeziehungen deutscher Städte und Kommunen im Bereich kommunaler Entwicklungszusammenarbeit bieten Heinz \& Langel (2002); für eine Systematisierung kommunaler Kooperationsbeziehungen aus internationaler Perspektive vgl. UN-HABIT AT \& WACLAC (2003).

2 Wie beispielsweise kommunale Politik und Verwaltung, Bildungseinrichtungen, Nichtregierungsorganisationen (NROs), örtliche Unternehmen u.a. 3 Brouns, Ott \& Scherhorn (2002), S. 14.

4 Ebd.

5 Bezüglich dieser sowie der weiteren Ausführungen muss einschränkend eingeräumt werden, dass es sich hierbei durchweg um Optionen handelt. Eine Untersuchung bezüglich der Realisierung dieser Optionen steht bislang noch aus. Mit der Ausarbeitung dieser und anderer Fragestellungen zum informellen Lernen in kommunalen Kooperationen zwischen Nord und Süd beschäftigt sich die Autorin dieses Beitrages in ihrer Dissertation.

6 Als allgemeines Ziel einer Bildung für nachhaltige Entwicklung schlagen de Haan \& Harenberg (1999, S. 62) den Erwerb von ,Gestaltungskompetenz vor. Diese bezeichnet das Vermögen, „die Zukunft von Sozietäten, in denen man lebt, in aktiver Teilhabe im Sinne nachhaltiger Entwicklung modif zieren und modellieren zu können." Für eine weitere Ausdifferenzierung von Gestaltungskompetenz als mehrdimensionale Kompetenzstruktur' vgl. de Haan \& Seitz (2001).

7 Europäische Kommission (2001), S. 33.

8 So beispielsweise im Zuge der konkreten Kooperation mit lokalen Partnern, Partizipation in lokalen Gremien, der Planung, Gestaltung und Oganisation von Partnerschaftsaktivitäten, Öffentlichkeitsarbeit u.ä.

9 Auf die soziokulturelle Einbettung informeller Lernorte, durch welche kulturelle Normen, Gedanken und Praktiken perpetuiert werden, macht Duensing (2006) am Beispiel von, Informal Science Centers' aufmerksam. Diese seien, ebenso wie formale Bildungsinstitutionen, als Produkte (,creations') der jeweiligen Kultur zu verstehen. 
10 Vgl. hierzu auch Schugurensky (2006), S. 168f.: „Although it may sound like a cliché, it is no less true that one of the best ways to learn democracy is by doing it, and one of the best ways to develop efective civic and political skills is by observing them in the real world and exercising them."

11 Vgl. auch Marsick \& Watkins (1990, S. 6): Informelles und inzidentelles Lernen trete häufg genau dann auf, ,,when the procedures and responses that people normally use fail. In such cases, people may become aware of many tacit, hidden, taken-for -granted assumptions. In the process of doing this, people often reframe the problem they are experiencing, that is, they realize that a particular situation can be de f ned and solved in many different ways."

12 Hierbei handelt es sich um eine gängige und vielfach vorzfindende Praxis in kommunalen Kooperationen.

13 Davon zeugen diverse Erfahrungsberichte sowie Interviews mitAkteuren, wie sie auch von derAutorin dieses Beitrages im Rahmen ihrer Dissertation geführt wurden.

14 Vgl. exemplarisch Johnson \& Wilson (2006) sowie Byrne \& Vincent (2004).

15 Und zwar sowohl intra- als auch interkommunal. Aus Gründen der Übersichtlichkeit wurden bislang Differenzen in der interkommunalen Kooperation besonders hervorgehoben. Aber auch innerhalb der jeweiligen Kommunen sind Differenzen anzunehmen, so bspw. zwischen Verwaltung und Wirtschaf bzw. NRO's etc. Aus einer solchen Perspektive würden dann - gegenüber grundlegenden interkommunalen Differenzen - Gemeinsamkeiten der kommunalen Akteure in Nord und Süd (so z.B. der Verwaltungen) stärker in den Blick rücken.

16 Auch Johnson \& Wilson (2006, S. 73) betonen - neben einer eher skeptischen Perspektive - die in der Differenz liegenden Optionen: „In the ideal view, difference is a driver of mutuality. (...) Mutuality, in this ideal sense, makes a virtue of difference, enabling each partner to offer and gain something. Importantly, it of fers an opportunity for learning." Lernend seien - unter bestimmten Voraussetzungen - letztlich auch Veränderungen der Bedingungen möglich: „,(..) partnerships conceived as learning model that build on mutuality and difference offer the potential to challenge power relations" (ebd., S. 71)

17 Hinsichtlich der Kompetenzen und Fähigkeiten im Sinne einer Bildung für nachhaltige Entwicklung - als Ausdifferenzierung von ,Gestaltungskompetenz' - beziehe ich mich im Folgenden auf de Haan \& Seitz (2001).

\section{Literatur}

Brouns, B./Ott, H. E./Scherhorn, G. (2002): Leitbilder und Ziele einer nachhaltigen Entwicklung in internationalen, europäischen und nationalen Zielf ndungsprozessen. Wuppertal. URL: http://www.agenda21nrw.de/download/globale_verantwortung.pdf (22.08.2007).
Byrne, A./Vincent, R. (2004): Learning in Partnerships. BOND / Exchange. London. URL: http://www .bond.org.uk/pubs/briefs/nslearning. pdf (22.08.07)

Duensing, S. (2006): Culture matters. Informal Science Centers and Cultural Contexts. In: Bekerman, Z. et al. (Hg.): Learning in places. The informal education reader. Bern, Berlin: Peter Lang. S. 183 - 202.

Europäische Kommission (2001): Mitteilung der Kommission: Einen europäischen Raum des Lebenslangen Lernens schaf fen. Brüssel. URL: http://ec.europa.eu/education/policies/1ll/life/communication/com_de.pdf (29.08.07).

Haan, G. de/Harenberg, D. (1999): Bildung für eine nachhaltige Entwicklung. Materialien zur Bildungsplanung und Forschungsförderung, H. 72. Bonn. URL: http://www.blk-info.de/f leadmin/BLK-Materialien/Heft72. pdf (30.08.07).

Haan, G. de/Seitz, K. (2001): Kriterien für die Umsetzung eines internationalen Bildungsauftrages. Bildung für eine nachhaltige Entwicklung. Teil 1 \& 2. In: Zeitschrift „21“-Das Leben gestalten lernen. Heft 01/2001 \& Heft 02/2001. München. URL: http://www .transfer-21.de/daten/texte/bildungsauftrag.pdf (30.08.07).

Heinz, W./Langel, N. (2002): Kommunale Entwicklungspartnerschaften - Kooperationsbeziehungen zwischen deutschen Städten und Kommunen in Entwicklungsländern, Transformationsstaaten und derVR China. Deutsches Institut für Urbanistik. Berlin.

Johnson, H./Wilson, G. (2006): North-South/South-North Partnerships: Closing The ,Mutuality Gap'. In: Public Administration and Development, 26, S. $71-80$

Marsick, V./Watkins, K. (1990): Informal and Incidental Learning in the Workplace. London.

United Nations Human Settlements Programme (UN-HABITAT)/World Association of Cities and Local Authorities Coordination (WACLAC) (2003): Partnership for Local Capacity Development. Building on the Experiences of City-to-City Cooperation. Nairobi. URL: http:/wwwunhabitat. org/pmss/getElectronicVersion.asp?nr=1187\&alt=1 (29.08.2007).

Schugurensky, D. (2006): „This is our school of citizenship”- Informal learning in local democracy. In: Bekerman, Z. et al. (Hg.): Learning in places. The informal education reader. Bern, Berlin: Peter Lang, S. $163-182$.

Dipl.-Päd. Ulrike Devers-Kanoglu, Jg. 1974, wissenschaftliche Mitarbeiterin am Lehrstuhl für Erziehungswissenschaft/Erwachsenenbildung undWeiterbildung der Universität zu Köln; Arbeits- und Forschungsschwerpunkte in den Themenbereichen Erwachsenenbildung, Informelles Lernen, Bildung für nachhaltige Entwicklung, Internationale Zusammenarbeit/Entwicklungszusammenarbeit mit dem Schwerpunkt kommunale Kooperationen, Globalisierung, Zivilgesellschaft und Bürgerbeteiligung. Mitglied der Arbeitsgruppe Informelles Lernen der UN-Dekade Bildung für nachhaltige Entwicklung. 